\title{
IMMERSING BRANCHED SURFACES IN DIMENSION THREE
}

\author{
JOE CHRISTY
}

(Communicated by Frederick R. Cohen)

\begin{abstract}
We present necessary and sufficient conditions for immersing and embedding branched surfaces in dimension three. We illustrate the result with some applications to dynamics. Finally, we discuss the extension to higher dimensions and codimensions.
\end{abstract}

\section{INTRODUCTION}

Branched surfaces have come under scrutiny lately in connection with the dynamics of expanding attractors and the topology of three-manifolds, see for example [W, O, C, GO]. Whereas in topology a branched surface is a tool for studying an ambient three-manifold and comes equipped with a canonical fibered neighborhood, in dynamics it is often interesting to study an attractor, and hence its associated branched manifold, such as a ding im sich, and we are faced with the question of when can it arise in the dynamics of some particular phase space. Remarkably enough, unlike the cases of ordinary surfaces in threemanifolds or branched one-manifolds in surfaces, there are branched surfaces that admit no embedding in any three-manifold. In Theorem 2.1 we give necessary and sufficient conditions for a branched surface to have an immersion in some three-manifold. Our condition is posed in terms of certain combinatorial data that determine a normal line bundle to the branched surface, and thus constructs a three-manifold into which the branched surface actually embeds. While our presentation is geometric, the algebraically inclined reader will realize that we are essentially computing when the first Stiefel-Whitney class of the branched surface vanishes. We will illustrate this with some examples that arose from our previous study of the dynamics of flows in dimension three. Finally, we will make some remarks about the situation in higher codimension.

\section{Definitions AND TERMinOlogy}

Definition 1.1. A branched surface, $\mathbf{W}$, is a two-dimensional complex that is locally homeomorphic, via orientation preserving charts $\phi$, to a neighborhood on one of the following two models, see Figure 1.1 on next page. Furthermore,

Received by the editors December 17, 1990.

1980 Mathematics Subject Classification (1985 Revision). Primary 57M20, 57N10, 58F12.

Key words and phrases. Branched surface, swaddled graph.

Supported in part by NSF grant DMS 8610730 (1). 

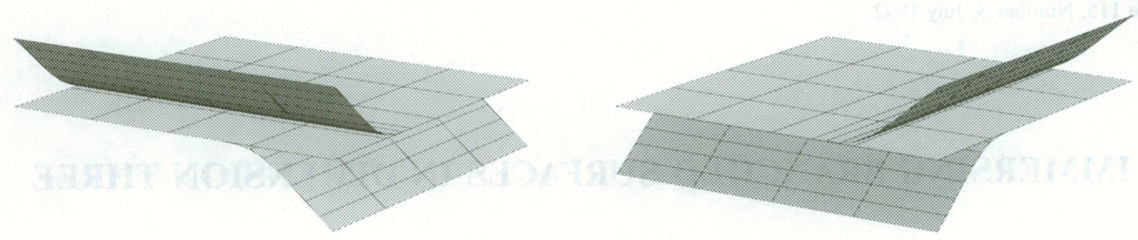

FIGURE 1.1. Left and right crossings.

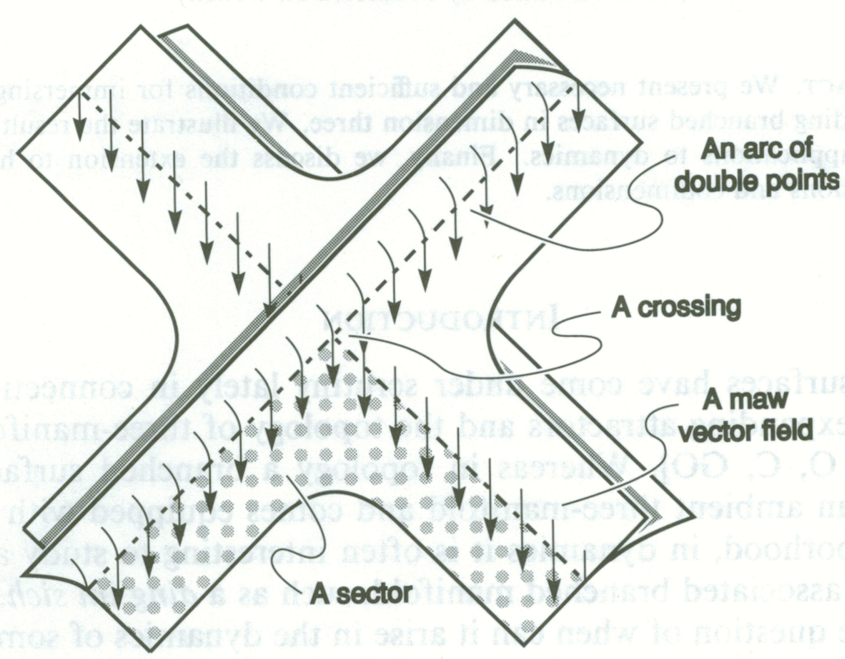

FIGURE 1.2. A piece of $\mathscr{Y}$ and some of its parts.

the transition maps for these charts are required to be smooth.

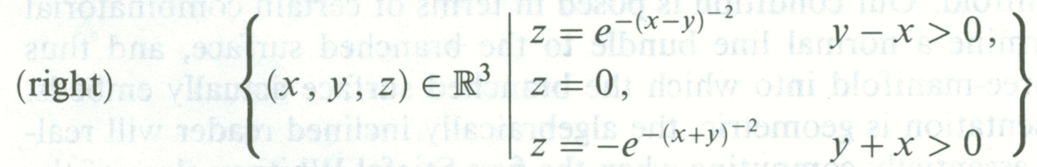

or

(left)

$$
\left\{\begin{array}{l|l}
(x, y, z) \in \mathbb{R}^{3} & \begin{array}{ll}
z=e^{-(x+y)^{-2}} & y+x>0, \\
z=0, & y-x>0, \\
z=-e^{-(x-y)^{-2}} & y
\end{array}
\end{array}\right\} .
$$

We call points sent by $\phi$ to $(0,0,0)$ crossings, and the set of points sent by $\phi$ to the rays $x= \pm y, x \neq 0$, arcs of double points. Together they form the branch locus, $\mathbf{B}$, of $\mathbf{W}$. When $\mathbf{W}$ is compact, $\mathbf{B}$ will be a union of smooth circles with normal crossings. We will denote by $\mathscr{Y}$ a small, smooth, closed regular neighborhood of $\mathbf{B}$ in $\mathbf{W}$. We will refer to the components of $\mathbf{W} \backslash \operatorname{int}(\mathscr{y})$ as the sectors of $\mathbf{w}$. On $\mathscr{Y}$ there is a natural homotopy class of nonsingular tangent vector fields, namely, those that are everywhere transverse to $\mathbf{B}$ and point from the locally two-sheeted side to the one-sheeted side of $\mathbf{B}$ in $\mathbf{W}$. We will call any such vector field a maw vector field. See Figure 1.2. In Figure 1.1 we are looking down the maw. 


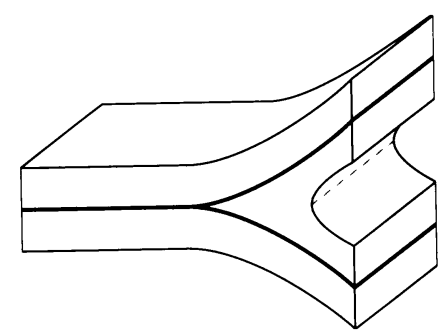

Figure 1.3. A fibered neighborhood of an arc of double points.

Of course, if a branched surface is to smoothly immerse in a three-manifold, it must have a tangent plane and hence a complementary normal line bundle.

Definition 1.2. A normal line bundle $\nu$ to a branched surface $\mathbf{W}$ is a space locally diffeomorphic to the $\mathbb{R}$ bundle spanned by $\partial / \partial z$ over one of the local models above, with the transition maps for these charts preserving the vertical direction.

Just as an embedded submanifold has a tubular neighborhood diffeomorphic to its normal bundle, similar reasoning constructs a fibered neighborhood of a branched surface embedded in a three-manifold.

Definition 1.3. A fibered neighborhood of a branched surface in a three-manifold is an open submanifold containing the branched surface that is locally diffeomorphic to a neighborhood in $\mathbb{R}^{3}$ of one of the local models above, fibered by segments parallel to the $z$-axis, with transition maps preserving the fibers. See Figure 1.3.

In [C] we studied the structure of branched surfaces by decomposing them into their sectors and the neighborhood $\mathscr{Y}$ of their branch loci. We showed that the structure of the normal line bundle to a branched surface $\mathbf{w}$ over $\mathscr{Y}$ is uniquely determined by the topology of $\mathbf{B}$, which in turn is determined by some combinatorial data, as follows.

Definition 1.4. A swaddled graph $\mathbf{B}_{s}$ is a 4-valent graph $\mathbf{B}$, together with the following additional data (see Figures 1.4 and 1.5 on next page):

(1) an embedding of a neighborhood of each vertex into a neighborhood of the origin in the plane, and an $\mathrm{R}$ or an $\mathrm{L}$ in one of the quadrants that this embedding determines,

(2) M's on some of the edges outside of a maximal tree.

Here $\mathbf{B}_{s}$ is an abstract model of a branch locus. We think of the embedding in (1) as given by the restriction to $\mathbf{B}$ of the inverse of the exponential map from the tangent bundle to $\mathbf{W}$; the label goes in the quadrant where $\mathbf{W}$ locally has three sheets, and the label itself indicates whether the crossing corresponding to the vertex has a right- or left-handed local model. The planar embedding near the vertices partitions the incident edges into two pairs that meet smoothly to form two transverse arcs, one from northwest to southeast, and the other from southwest to northeast; in a right-hand crossing we refer to the SW-NE arc as the upper arc and the NW-SE arc as the lower arc, in a left-hand crossing the roles are reversed. Globally this decomposes $\mathbf{B}_{s}$ into smooth circles with two 

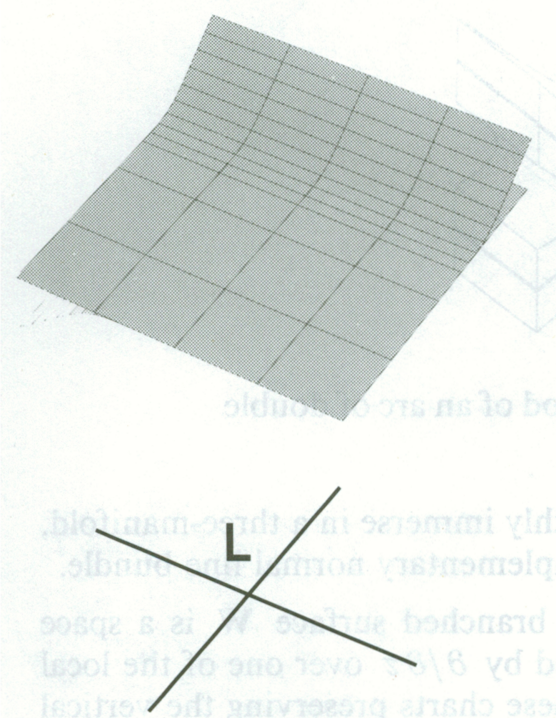
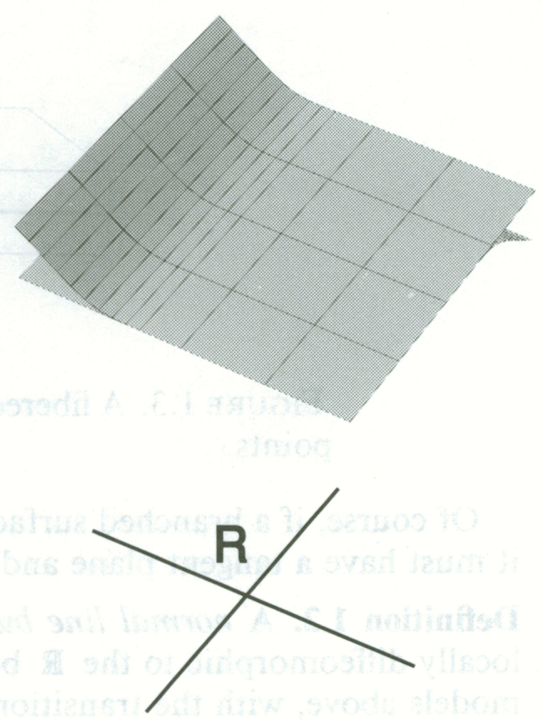

FIGURE 1.4. Swaddling vertices of $\mathbf{B}$.
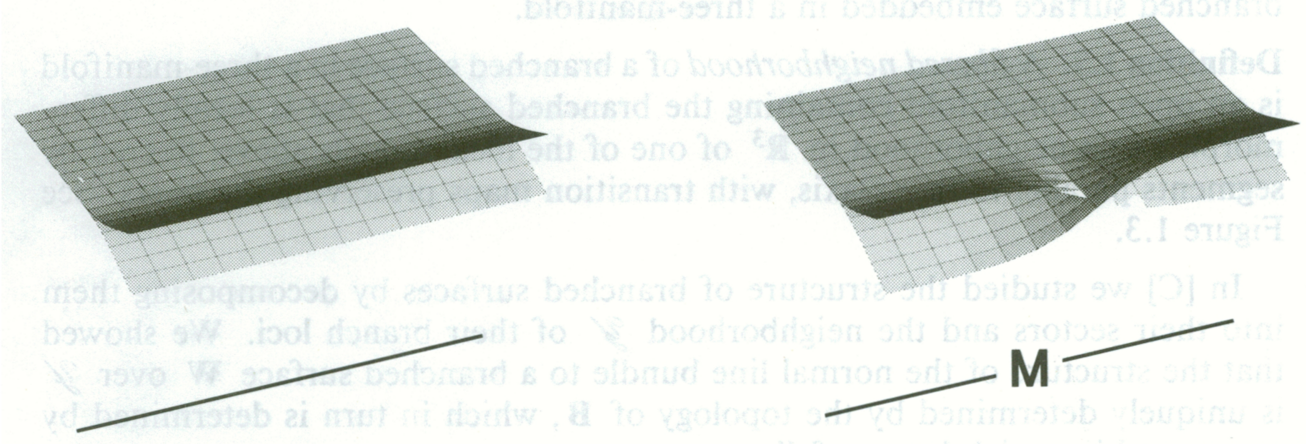

FIGURE 1.5. Swaddling edges of $\mathbf{B}$.

points identified at each crossing. We can also construct a maw vector field locally near the crossing as any extension by a vector field $\mu$, transverse to both smooth arcs of $\mathbf{B}_{s}$, of a vector $v$ at the crossing that points from the labeled quadrant to the opposite quadrant. Along each edge of $\mathbf{B}_{s}$, there are two essentially different extensions of the tangent bundle to $\mathbf{W}$ near the crossings; only one of these allows a nonsingular extension of $\mu$. Thus we can reconstruct $\mathscr{Y}$, and uniquely distinguish the locally two-sheeted side from the locally onesheeted side along the edges of $\mathbf{B}$.

Once we choose a particular Lipschitz maw vector field $\mu$, the boundary of $\mathscr{Y}$ on the locally two-sheeted side has a natural structure as a $\mathbf{S}^{0}$-bundle over $\mathbf{B}$ away from the crossings: the fiber over a point $b$ of $\mathbf{B}$ consists of the two points in the boundary of $\mathscr{Y}$ that flow into the maw to $b$. There are three points in $\partial \mathscr{Y}$ that flow toward each crossing. Examining Figure 1.2, it should be clear how choosing two arcs in $\mathscr{Y}$, each with an endpoint at one of the three points 
in $\partial \mathscr{Y}$ that flow toward the crossing and parallel to one of the smooth arcs in $\mathbf{B}_{s}$ allows the construction, via the $\mu$-flow, of the bundle structure to extend to the smooth circles in $\mathbf{B}_{s}$. In this extension, one point from each of the fibers over the two points $c_{u}$ and $c_{l}$ of the smooth circles which are identified at a crossing $c$ are themselves identified in descending from the bundle over the smooth circles to $\partial \mathscr{Y}$.

Thinking of $\mathbf{S}^{0}$ as $\mathbb{Z} / 2$, we label one of the points in an ordinary fiber 0 and the other 1, agreeing to do this labeling continuously and consistently with the requirement that the point 1 in the fiber over the lower arc be identified with the point 0 in the fiber over the upper arc so that we can speak sensibly of the global structure of this bundle. Once we have chosen a maximal tree in $\mathbf{B}_{s}$, the remaining edges generate the fundamental group of $\mathscr{Y}$, and the labels M, for mirror, in (2) pick out those edges for which the holonomy of the $\mathbf{S}^{0}$ bundle around the corresponding element of $\pi_{1}$ is orientation reversing; in other words, the holonomy of the equivalent $\mathbb{Z} / 2$ principal bundle is non trivial.

When we have an immersion of $\mathbf{W}$ into some manifold, the $\mathbf{S}^{0}$-bundle above is canonically bundle homotopic to the boundary of an interval subbundle of the normal bundle to the immersion restricted to $\mathbf{B}$; the fiber of this bundle is tangent to a copy, homotoped out into the ambient manifold so as to be normal to $\mathscr{Y}$, of the $\mu$ orbit in $\mathscr{Y}$ from the 0 point of the $\mathbf{S}^{0}$ fiber, to the base point $b$ on $\mathbf{B}$, followed by the time reversal of the $\mu$ orbit from the 1 point of the fiber to $b$. In particular, when we have an immersion in codimension 1 , this interval subbundle determines the normal line bundle. As we argued in [C], since $\mathscr{Y}$ retracts onto $\mathbf{B}$, which is a $K(\pi, 1)$, and there are precisely two homotopy classes of homeomorphisms of $I$ distinguished by their action on the orientation, there is a unique homotopy class of interval bundles over $\mathscr{Y}$ consistent with the $\mathbf{S}^{0}$-bundle over $\mathbf{B}$.

In [C] we were concerned with the restricted subclass of branched surfaces that come from hyperbolic attractors in flows, and there was a third class of data in the definition of swaddled graph, which served to specify how the sectors were attached to $\mathscr{Y}$; since we are now considering branched surfaces in general, we will not require this further data in the present definition.

\section{IN THE HALL OF MIRRORS}

Given a branched surface, as we traverse a boundary component of a sector we will label it with mirrors as we pass by mirrors in the branch locus at the core of that portion of $\mathscr{Y}$ upon which it is incident.

Theorem 2.1. A branched surface has a normal line bundle, and hence admits an immersion in codimension 1 , if and only if the boundary of each sector contains an even number of mirrors.

Proof. We saw earlier that the swaddling of $\mathbf{B}$ gives a unique normal line bundle over $\mathscr{Y}$, so we need only ascertain when this bundle extends over the sectors. We treat each sector individually.

Since connected surfaces with boundary are also $K(\pi, 1)$ 's, the argument above shows that every line bundle over a connected surface with boundary determines and is uniquely determined, up to homotopy, by a $\mathbf{S}^{0}$-bundle, the boundary of its unit interval subbundle. In light of this reduction, our theorem is equivalent to the assertions that a $\mathbf{S}^{0}$-bundle over a surface with boundary, 
when restricted to the boundary, always gives rise to an even number of nontrivial bundles, and conversely, whenever there are $\mathbf{S}^{0}$-bundles over the boundary, an even number of which are nontrivial, there is an extension over the interior.

Suppose that there were a sector, $\Sigma$, with an odd number of boundary components for which the holonomy was nontrivial. The holonomy of a $\mathbf{S}^{0}$-bundle over $\Sigma$ gives rise to a homomorphism from $\pi_{1}(\Sigma)$ to $\mathbb{Z} / 2$, in other words, a cohomology class $\gamma$ in $H^{1}(\Sigma ; \mathbb{Z} / 2)$. By restriction this gives rise to a class $\eta$ in $H^{1}(\partial \Sigma ; \mathbb{Z} / 2)$; our supposition is that $\eta([\partial \Sigma]) \neq 0$. On the other hand, consider the tail of the long exact cohomology sequence

$$
\cdots \rightarrow H^{1}(\Sigma ; \mathbb{Z} / 2) \stackrel{i^{*}}{\longrightarrow} H^{1}(\partial \Sigma ; \mathbb{Z} / 2) \stackrel{\delta^{*}}{\longrightarrow} H^{2}(\Sigma, \partial \Sigma ; \mathbb{Z} / 2) \longrightarrow 0 .
$$

Now $\delta^{*}\left(i^{*}(\gamma)\right)=\delta^{*}(\eta)=0 \in H^{2}(\Sigma, \partial \Sigma ; \mathbb{Z} / 2)$, so $\eta([\partial \Sigma])=0$, a contradiction.

For the converse, suppose that we have a $\mathbf{S}^{0}$-bundle over the boundary of a sector $\Sigma$ with nontrivial holonomy over an even number of boundary components. Join these boundary components pairwise by disjoint embedded arcs in $\Sigma$, then cut $\Sigma$ apart along these arcs. Over the new surface $\Sigma_{0}$, erect the product $\mathbf{S}^{0}$-bundle. Now reassemble $\Sigma$ by identifying arcs in its boundary and in the $\mathbf{S}^{0}$-bundle identify the fibers with a flip. We are left with $\mathbf{S}^{0}$-bundle over $\Sigma$, which extends the original $\mathbf{S}^{0}$-bundle over the boundary.

Proposition 2.2. If a branched surface $\mathbf{W}$ admits a normal line bundle there is a unique fibered neighborhood consistent with this bundle structure, and hence $\mathbf{W}$ embeds in some three-manifold.

Proof. Away from the interior of $\mathscr{Y}$ we embed $\mathbf{W}$ as the zero section of the bundle. $\mathscr{Y}$ we embed by the charts $\psi$ into $\mathbb{R}^{3}$ fibered by vertical lines.

\section{EXAMPLES}

Example 3.1. The suspension of the doubling map $z \mapsto z^{2}$ on the circle

$$
\{z \mid\|z\|=1\} \text {. }
$$

Here the branch locus is a single circle, and there is one sector that is an annulus with one boundary glued to $\mathbf{B}$ by a degree one map and the other glued by a degree 2 map. In order to achieve the second gluing there must be a single mirror on $\mathbf{B}$, and thus three mirrors on the boundary of the sector; see Figure 3.1. In this context, Theorem 2.1 is just the observation that over the locally one-sheeted boundary of $\mathscr{Y}$, the normal line bundle is a Möbius band, while over the locally two-sheeted boundary we have split it along its core so we have a cylinder, and that there is no line bundle over the annulus having this structure over the boundaries.

Therefore, exfoliating this suspension as in [C], that is, taking the obvious constant speed flow on the suspension of the inverse limit of the original map, we get an attractor for a flow that is unrealizable in any three-manifold.

Example 3.2. The neighborhood $\mathscr{y}$ coming from the swaddled graph in Figure 3.2 has the proper boundary behavior to be completed, by capping the two boundary components with disc sectors, to a branched surface with $\chi=0$ and supporting a nonsingular semiflow [C]. 


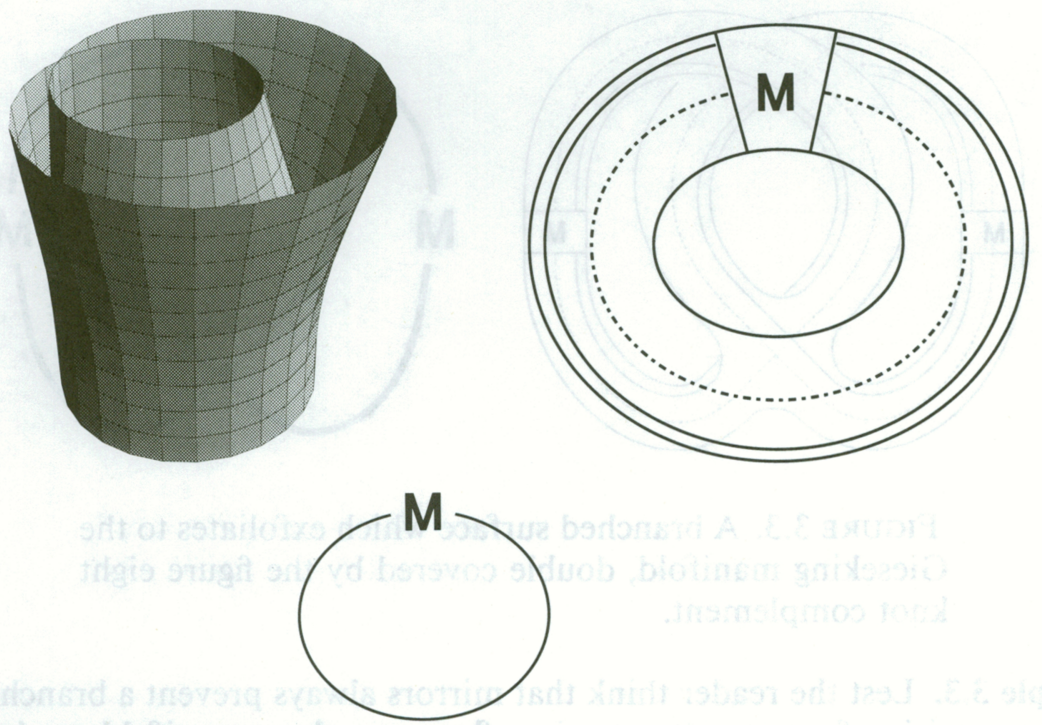

Figure 3.1. The Möbius Y-bundle: two views of a neighborhood of the branch locus of the suspension of the doubling map of the circle and the associated swaddled graph. This branched surface cannot be immersed in codimension 1 .

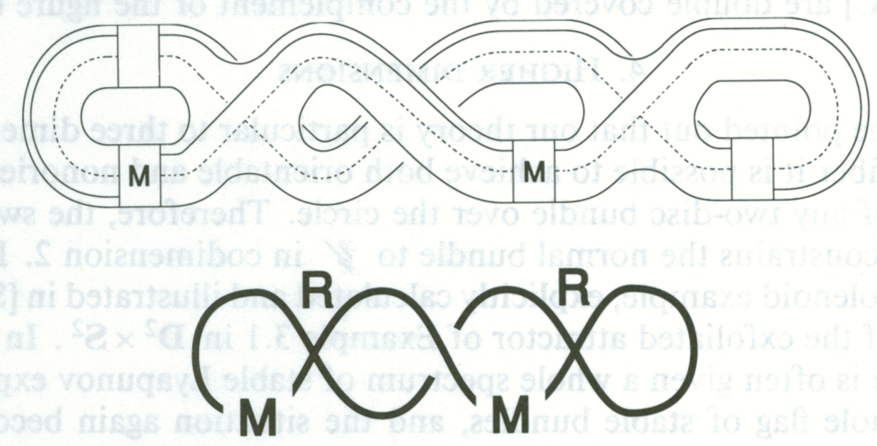

FIGURE 3.2. A swaddled graph that may be completed to a branched surface that admits a nonsingular semiflow or one that admits an immersion in codimension 1 , but not both. Note that the empty boxes correspond to half twists of $\mathscr{Y}$.

However, one readily checks that one boundary component of $\mathscr{Y}$ contains five mirrors, and the other contains one, so there can be no normal line bundle to this branched surface. On the other hand, if we create a branched surface of negative Euler characteristic by attaching a single sector with two boundary components, we will have six mirrors in the boundary so that the resulting branched surface will embed in some three-manifold. It will no longer support a nonsingular semiflow however, so we have another example of a swaddled graph that gives rise to an attractor unrealizable in dimension three. 

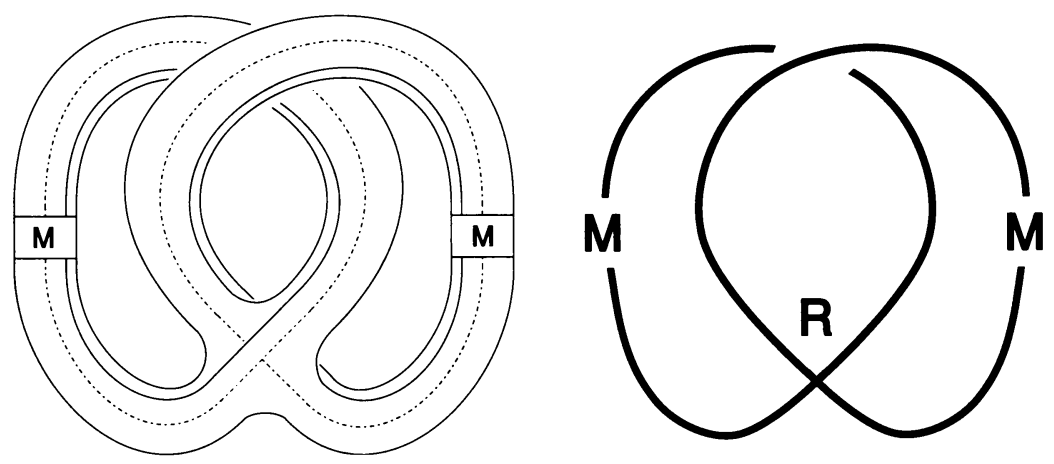

Figure 3.3. A branched surface which exfoliates to the Gieseking manifold, double covered by the figure eight knot complement.

Example 3.3. Lest the reader think that mirrors always prevent a branched surface from arising from an attractor in a flow on a three manifold, we have the swaddled graph in Figure 3.3. Its regular neighborhood $\mathscr{Y}$ has a single boundary component with six mirrors, so the reader can check that we may cap it off with a disc and get a dynamic branched surface as in [C], in other words, one that comes from an expanding attractor in a flow on a three-manifold. It is interesting to note that the three-manifolds in both this example and the last example in [C] are double covered by the complement of the figure eight knot.

\section{HighER DIMENSIONS}

It should be pointed out that our theory is particular to three dimensions. By rotating the fiber it is possible to achieve both orientable and nonorientable line subbundles of any two-disc bundle over the circle. Therefore, the swaddling of B no longer constrains the normal bundle to $\mathscr{Y}$ in codimension 2 . In fact, the well-known solenoid example, explicitly calculated and illustrated in [S], gives an embedding of the exfoliated attractor of Example 3.1 in $\mathbf{D}^{2} \times \mathbf{S}^{2}$. In dynamics, however, one is often given a whole spectrum of stable Lyapunov exponents, so there is a whole flag of stable bundles, and the situation again becomes more complicated since there would now be a sequence of codimension one problems.

\section{ACKNOWLEDGMENT}

It is a pleasure to thank David Gabai for posing, during a conversation about Example 3.1, the question of when does an arbitrary branched surface embed in dimension three and proposing the solution given here. I would also like to thank the Institute for Advanced Study for its hospitality during part of the preparation of this paper.

\section{REFERENCES}

[C] J. Christy, Branched surfaces and attractors I: dynamic branched surfaces, Trans. Amer. Math. Soc. (to appear).

[GO] D. Gabai and U. Oertel, Essential laminations in 3-manifolds, Ann. of Math. (2) 130 (1989), 41-73.

[O] U. Oertel, Incompressible branched surfaces, Invent. Math. 76 (1984), 385-410. 
[S] M. Shub, Global stability of dynamical systems, Springer-Verlag, New York, 1986.

[Sp] E. H. Spanier, Algebraic topology, McGraw-Hill, New York, 1966.

[W] R. F. Williams, Expanding attractors, Inst. Hautes Études Sci. Publ. Math. 43 (1973), 473-487.

Department of Mathematics, Boston University, Boston, Massachusetts 02215

Current address: Department of Mathematics, University of California, Santa Cruz, California 95064

E-mail address: joe@cats.ucsc.edu 